\title{
A design comparing free- and forced-trial alternation in the rat at two interchoice intervals*
}

\author{
SUSAN KARP MANNING \\ Humer College of the City lniversin of New York \\ Vew York Vew York 10021
}

A design is derived which equates the normally differing chance levels of alternation in the free-and forced-trial paradigms. Using 37 rats, this design was used to compare alternation levels in the two paradigms at two interchoice intervals. Forced-trial alternation was found to be significantly higher and longer lasting than free-trial alternation. It is suggested that the methodology presented here be considered by future researchers dealing with the two paradigms

Studies of spontaneous alternation, the above-chance probability of differing successive choices in a two-alternative situation, have used two paradigms. In the free-trial paradigm, both choices are free, while in the forced-trial paradigm, Choice 1 is predetermined and Choice 2 is free.

Although more alternation has generally occurred in the forced-vs the free-trial paradigm (Dember \& Fowler, 1959: Thompson, 1960), results have been inconsistent (Hughes, 1966). It is contended that previous studies are somewhat questionable because the chance probability of alternation (PA) differed for the paradigms and corrections were not made. A description of and solution to this problem is outlined here.

Define the S's two choices as $M$ and $O$ and the probability of choice of $\mathrm{M}$ as $\mathrm{m}$ and of $\mathrm{O}$ as $(1-\mathrm{m})$. Dember and Fowler's (1958) expression for PA for the free-trial paradigm may be written as Eq. 1.

$$
P A=m(1-m)+(1-m) m=2 m(1-m) .
$$

A complication described, although not handled. by Koppenaal (1962) is that the sample estimate of Eq. 1 is biased in the direction of underestimation. The bias is maximal when $\mathrm{m}=0.5$ and disappears entirely only when $\mathrm{m}=0.0$ or 1.0 . In obtaining an unbiased estimate of Eq. 1, m may be estimated by counting the number of $\mathrm{M}$ choices $(\mathrm{k})$ on independent trials and dividing by the total number of choice opportunities $(\mathrm{N})$. Because Eq. 1 is equal to twice the variance of the binomial distribution. multiplying by $\mathrm{N} / \mathrm{N}-1$ leads to the needed equation. The resulting estimator, which should be used when $\mathrm{N}$ is small, is

\footnotetext{
* This paper contains a part of a dissertation submitted to the Graduate School of the University of California, Riverside, in 1971. in partial fulfillment of the requirements for the PhD degree. I wish to thank my committee members. Austin $H$. Riesen and Sally Sperling, and $m y$ thesis chairman, Arlo $\mathrm{K}$. Myers, for their help. Address reprint requests to Susan Karp Manning. Department of Psychology. Hunter College. $695 \mathrm{Park}$ Avenue. New York. New York 10021 .
}

$$
2(\mathrm{k} / \mathrm{N})(1-\mathrm{k} / \mathrm{N})(\mathrm{N} / \mathrm{N}-1) .
$$

Koppenaal also described complications resulting from using the mean of individual Ss' preferences for M to calculate a group PA. If heterogeneous preferences exist. such a procedure will result in an overestimation of the group PA, which increases with the dispersion of the preferences. Past researchers have not considered Koppenaal's criticisms or the solutions to be presented here.

In addition, researchers assumed PA to be the same in the two paradigms (Dember \& Fowler, 1959: Hughes. 1966: Thompson. 1960). It has been shown that this is not the case (Manning, 1971). Define $f$ and $(1-f)$ as the proportion of responses that are forced toward $\mathrm{M}$ and $\mathrm{O}$. respectively. Thus, in the forced-trial paradigm,

$$
P A=f(1-m)+(1-f) m .
$$

The relationship between $\mathrm{PA}$ in the two paradigms may be quantified for a $S$ with a constant preference for $M$ through a quantity, E, obtained by subtracting Eq. 1 from Eq. 3.

$$
\begin{aligned}
& E=f(1-m)+(1-f) m-[2 m(1-m)] \\
& E=(f-m)(1-2 m) .
\end{aligned}
$$

When $E$ is not equal to zero, as is true for most values of $f$ and $m$. PA differs for the two paradigms. For example. if $m=0.9$ and $f=0.5 . E=.32$. With a $P A=.18$ for free trials and .50 for forced trials. it is clear that direct comparisons of alternation are invalid. Setting $E$ equal to zero leads to $f$ values of $m$ and 0.5 . Thus. when there are equal preferences for $\mathrm{M}$ and $\mathrm{O}$. or when the preference proportion is equal to the forcing proportion. PA is the same in the two paradigns.

None of the previous studies have met all the requirements outlined above. Furthermore, most designs comparing the paradigms $(f=0.5 . m \neq 0.5$. due to differentiated mazes) lead to higher PAs for the forced-trial paradigm (a positive value of $\mathrm{E}$ ) and bias the results.

In order to allow valid comparisons of alternation levels beiween the paradigms. the $\mathrm{m}=\mathrm{f}$ solution to $\mathrm{Eq} .4$ was used because obtaining an $m=0.5$ in a two-choice situation is difficult to assure experimentally. Each $S$ was forced toward $\mathrm{M}$ on a proportion of trials equal to its preference for $M$ on the first choice of the free trials. To avoid difficulties from using group means to estimate $M$ when individual preferences may vary. a separate value of $\mathrm{m}$ and a corresponding $\mathrm{PA}$ using the $\mathrm{N} N-1$ correction was calculated for each $\mathrm{S}$. 
An experiment was designed to compare the levels of alternation for the two paradigms and two different interchoice intervals (ICIs), using the method described here. In addition, this design tested previous findings which indicate the relatively long-lasting properties of forced-as contrasted with free-trial alternation (Dember \& Fowler, 1959).

\section{METHOD \\ Subjects}

The Ss were 48 male. 80-85-day-old Long-Evans hooded rats from Rockland Farms. They were on ad lib food and water throughout the study and were handled 4 to $5 \mathrm{~min}$ per day for 5 days preceding the experiment.

\section{Apparatus}

The apparatus was a plywood $T$-maze with 5 -in.-wide runways, a $16^{1}{ }_{2}$-in. maze stem, and $12 \frac{1}{2}$-in. goal arms. There were three manually operated retrace doors. One door divided the $83 / 4$-in. startbox from the remainder of the stem, while the other two doors were located at the goal arm entrances.

The interior of the left goal arm was painted black and that of the right arm was painted white. All other wooden surfaces of the maze were painted gray. A photoelectric cell was located 7 in. inside each goal arm. 2 in. above the floor.

The maze was covered with gray translucent plastic and hinged to a gray plastic floor, which allowed the maze to be lifted and the floor to be cleaned.

\section{Procedure}

On both choices of each trial, the $S$ was placed in the startbox for $5 \mathrm{sec}$. Then the retrace door was opened, automatically starting an electric timer. When the S entered 7 in. into one of the goal arms. the photobeam was interrupted and the timer stopped. indicating a choice. After the interruption of the photobeam, the retrace door was closed manually. The $S$ was retained in the goal arm for $10 \mathrm{sec}$ and then removed from the maze. Between choices, a damp sponge was used to clean the maze floor, reducing the formation of odor trail. The entire process was repeated for all Ss on all choices.

The $\mathrm{S} s$ were given one trial per day on 14 successive days. Each trial consisted of two choices. On odd-numbered days, the $S$ 's first choice was free; on even-numbered days, the S's first choice was forced by closing the door to one goal arm. The second choice of each day's trial was free.

On a forced trial, the direction of forcing was determined by the choice the $S$ made on the first choice of the free trial given the day before For example, a $S$ choosing white on the first choice of Day 5 would be forced white on Day 6 . This procedure served to equate $f$ to $k / N$, the estimate of $m$.

The Ss were divided randomly into two groups of 24 . The ICI of one group was $90 \mathrm{sec}$; the ICI for the other was $60 \mathrm{~min}$. For both groups, after $4 \mathrm{~min}$ elapsed on any given choice, Ss failing to trigger the electric eye were removed from the maze. Three such incidents eliminated a $\mathrm{S}$ from the experiment. Eleven $\mathrm{Ss}$ were eliminated, leaving $19 \mathrm{Ss}$ in the 60 -min group and 18 in the 90 -sec group.

\section{RESULTS}

An estimate of $m$ was obtained for each $S$ by computing $\mathrm{k} / \mathrm{N}$ on the first choice of each free trial $(\mathrm{N}=7)$. This value was substituted in Eq. 2 to calculate PA for each $\mathrm{S}$ individually. Deviation scores for both free and forced trials were obtained for each $S$ by subtracting the number of expected alternations from the number observed.

A two-way analysis of variance was computed using the deviation scores in order to determine whether significant differences in alternation level were present between free and forced trials and between the $90-\mathrm{sec}$ and $60-\mathrm{min}$ ICIs. The trials main effect was significant. $F(1,35)=5.88, p<.025$. Neither the ICI main effect nor the Trials by ICI interaction were reliable, $F(1,35)=$ .38 and $F(1,35)=.82$ : both $p s>.05$. Forced trials lead to higher levels of alternation than do free trials, as found by previous researchers. However, the differences between alternation levels for the ICIs did not reach significance.

The deviation scores used in the analysis of variance were also used to test for the presence of spontaneous alternation at the two ICIs. For each condition, a one-sample $t$ test was used to test the null hypothesis that the mean difference between observed and expected alternation was zero.

For the free-trial conditions, the $90-\mathrm{sec}$ group evidenced significant spontaneous alternation (mean deviation $\left.=.53, \sigma_{\mathrm{D}}=.26\right), \mathrm{t}(17)=2.04, \mathrm{p}<.05$, while the 60-min group did not (mean deviation $=.15, \sigma_{\mathrm{D}}=$ $.35), t(18)=.42, p>.05$. For the forced-trial groups, spontaneous alternation was present for both the 90 -sec group (mean deviation $=1.11, \sigma_{\mathrm{D}}=.41$ ), $\mathrm{t}(17)=2.71$. $\mathrm{p}<.01$, and the $60-\mathrm{min}$ group (mean deviation $=.95$, $\left.\sigma_{\mathrm{D}}=.37\right), \mathrm{t}(18)=2.57, \mathrm{p}<.01$. These results were consistent with the previously obtained longer lasting properties of forced-trial alternation as compared with free-trial alternation.

\section{DISCUSSION}

The comparisons between alternation levels in the free- and forced-trial paradigms indicate spontaneous alternation to be significantly higher after forced than after free trials. This appears to be the first study with rats to employ a design where the PA is the same under both conditions. Thus, although previous work pointed to this result, the current research is the first to demonstrate the finding conclusively.

The insignificant differences in alternation level for the two ICIs were not expected. However, the results of the thests were consistent with the previous finding that forced-trial alternation is longer lasting than free-trial alternation. Experimentation on forced-trial alternation to determine (a) the actual duration of the alternation tendency and (b) the cause of this long duration would be of interest.

Finally, and most important, it is suggested that the methodology used in this experiment be considered by researchers comparing free and forced-trial alternation. It should be added that researchers dealing with the free- or the forced-trial paradigm alone should also consider using this analysis since preferences for $m$ are likely to vary among. $g_{i}$ s or among values of the independent variable. It is essential, if the presence of spontaneous alternation is to be ascertained, that the true PA be known. In addition, if comparisons of alternation levels are to be made, it is necessary that appropriate corrections be made for potential differences in PA. This applies within the two paradigms as well as between them.

\section{REFERENCES}

Dember, W. N., Fowler, H. F. Spontaneous alternation behavior. Psychological Bulletin, 1958, 55, 412-426.

Dember, W. N., \& Fowler, H. F. Spontaneous alternation after free and forced trials. Canadian Journal of Psychology, 1959. 13, 151-154. 
Hughes, R. N. Stimulus change effects of forcing in spontaneous alternation behavior. Psychological Reports, 1966, 19 , 515-518.

Koppenaal, R. J. On the determination of expected probabilities of alternation. Psychological Reports, 1962, 11, 666 .

Manning, S. K. A method for assessing the chance level of alternation in the forced-trial paradigm. American Journal of
Psychology, 1971, 84, 421-424.

Thompson, M. E. Alternation in a $T$-maze as a function of three variables. Psychological Reports, 1960, 7, 103-110.

(Received for publication July 24, 1972; revision accepted February 18, 1973.) 